\title{
Computer Aided Planning and Execution of Craniofacial Surgical Procedures
}

\author{
Court Cutting, M.D., ${ }^{*}$ Russell Taylor, Ph.D., ${ }^{* *}$ Fred Bookstein, Ph.D., ${ }^{* * *}$ \\ Deljou Khorramabadi, M.S.,* Betsy Haddad, M.S.,* Alan Kalvin, Ph. D.,** \\ Hiechun Kim, B.S., ${ }^{*}$ and Marilyn Noz, Ph.D.****
}

\begin{abstract}
*Institute of Reconstructive Plastic Surgery, New York University Medical Center, New York, New York

**IBM Corporation, Watson Research Center, Elmsford, New York

***Center for Human Growth \& Development, University of Michigan, Ann Arbor, Michigan

****Department of Radiology, New York University Medical Center, New York, New York
\end{abstract}

In 1983 we began clinical evaluation of a surgical simulation program based on a threedimensionalization of a cephalogram (Cutting et al. 1986a). Two orthogonal X-ray views are used to locate 34 points on the face. A simple wire frame visual representation was used to perform an interactive three-dimensional surgical simulation. As a large database of such X-rays already existed (Grayson 1988 et al), results could be compared with an age, race and sex appropriated normal form. Optimization routines were added which had been created. Numerical printouts specified the movements of each piece. The surgeon could use these "surgical blueprints" in the operating room.

Eighteen months later we developed a custom, CT based surgical simulation program (Cutting et al. 1986). The first attempt at CT based surgical simulation used a commercial $\mathrm{CAD}$ package (Marsh et al 1985). At present a number of programs are available designed specifically for use by surgeons (Udupa \& Odhner 1990 \& Cutting 1991). We expected these programs to replace the earlier cephalometric wireframe program given the much richer data set that CT provides. After five years experience we found the CT based program to be much less useful than the cephalometric one. The critical factor was automated positioning of the bone fragments to best fit a normal form. The CT based program was useful only as an artist's tool, but these artistic maneuvers were done well in the mind of the surgeon. Quantitation was missing.

In 1985 we began developing software which would perform a quantitative, bone fragment positioning, CT based surgical simulation package with some form of numerical report which could be used in the operating room. We began with $0-7803-0785-2 / 92 \$ 03.00$ @IEEE the development of a high quality surface segmentation program which stored the data in a topologically connected form (Kalvin 1991). The connectivity allowed software to be written which would semiautomatically locate features on the skull such as ridges and landmarks which capitalized on the efficiency of nearest neighbor search.

Generic software was then written which took surface CT data and a standard "mapfile" which described the expected structure of the object. The mapfile guides a technician through semiautomated localization of the features on the object. Ridge curves on the surface of the skull are the primary features used. Curvature maxima on these ridges are used as landmarks. Geodesics connecting landmarks allow the skull surface to be decomposed into standard patches. Each point on the skull is thereby assigned a standard parametric identity (Bookstein \& Cutting 1988).

We need "averages" to use as criterion in these surgical simulations. Statistically, an average can be constructed as a derived form which has the minimum summed squared distance to each of the forms of a sample; then the nature of the average is a function of the formula we choose to represent "distance". In accordance with the principles of modern multivariate morphometrics (Bookstein, 1992), we constrain "distance" to be consistent with the biological labelling given by the mapfile. In a first step, the landmark configurations are averaged in the appropriate linearization of Kendall's shape space. This step is least-squares in so-called Procrustes distance (summed squared distance 
between points at the superposition minimizing that distance). For the remaining steps, the precision of the average can be increased if we first normalize each landmark configuration to this average "polyhedron". In a second step, curves linking pairs of landmarks (curvilinear edges of the polyhedron formed by the landmarks) are averaged perpendicular to their chords using a third step, ordinary points of surface patches inside these boundary curves are averaged along fibers normal to a parametric representation of a "standard" lofting scheme (which takes the anisotropic surface curvatures along ridge curves into account). In the actual surgical simulation, the computed "optimum" is itself a function of a subjectively assigned weighting among these three candidate distance functions. Different operations attending to different parts of the skull entail different weight profiles; for instance, while a simple orthognathic procedure can be optimized mostly by reference to conventional dentofacial point landmarks, a LeFort III requires attention to the ridge curves on the cheekbones, and a forehead advancement requires emphasis upon the approach to average of the ordinary surface points of the forehead.

The raw data for all these steps in the averaging come from CT scans of a collection of skulls from the Cleveland Museum of Natural History. The distance functions here permit an immediate extension of the multivariate analysis to the usual tactics of multivariate morphometrics: descriptions of within-group variability, computation of a customized surgical goal that adjusts for statistical expectations of relapse or growth remaining, simulation minimizing a robust Mahalanobis (covariance-based) distance instead of the geometric ones reviewed here, etc. The incommensurability of the three functions is no obstacle to these statistical maneuvers (Bookstein, 1992).

Quantitative execution of the operative derived as described above was designed by one of us (RHT) with consultation with another (CBC) (Taylor et al, $1991 \mathrm{a} \& \mathrm{~b})$. High precision planning is of little use if the surgeon is unable to carry it out. Cutting the bone fragments and fixing them into rigid fixation with screws and plates is done quite well by a human surgeon with currently available technologies. After cutting the fragment, performing the designed rigid motion relative to the skull base is not done accurately at all by the human surgeon. An optical system which tracks the position of diode beacons attached to the fragment, measures the rigid motion precisely. A passive manipulator with six degrees of orthogonally decouple freedom is used to position the fragment. Feedback to the surgeon from the optical sensor is provided to the surgeon on a workstation in the operating room which allows him/her to adjust the the manipulator appropriately. This system is currently undergoing bench testing before proceeding with clinical trials.

\section{BIBLIOGRAPHY}

Bookstein, F.: Morphometric Tools for Landmark Data. Cambridge University Press, 1991.

Bookstein, F., \& Cutting, C.: A proposal for the apprehension of curving craniofacial form in three dimensions. In Vig, $\mathrm{K}$. and Burdi, A.(eds.) Craniofaical Morphogenesis and Dysmorphogenesis. pp 127-40, Monograph of the Center for Human Growth and Development: Ann Arbor Michigan 1988.

Cutting, C., Bookstein, F., Grayson, B., Fellingham, L., and McCarthy, J.: Three-dimensional computer assisted design of craniofacial surgical procedures: Optimization and interaction with cephalometric and CT-based models. Plast. Reconstr. Surg. 77:877, 1986a.

Cutting, C., Grayson, B., Bookstein, F., Fellingham, L., and McCarthy, J.: Computer-aided planning and evaluation of facial and orthognathic surgery. Clin. Plast. Surg. 13:449, 1986b.

Cutting, C.: Applications of computer graphics to the evaluation and treatment of major craniofacial malformations. In Udupa, J. and Herman, G.(eds.) 3D Imaging in Medicine pp 163-189, Boca Raton: CRC Press, 1991.

Grayson, B., Cutting, C., Bookstein, F., Kim, H., and McCarthy, J.: The three-dimensional cephalogram: Theory, technique, and clinical application. Am. J. Orthod. Dentof. Orthop. 94:327, 1988.

Kalvin, A.: Segmentation and Surface-based Modelling of Objects in 3D Biomedical Images. Ph.D. Thesis, New York University, New York, 1991.

Marsh, J., Vannier, M., Stevens, W., Warren, J., Gayou, D., and Dye, D.: Computerized Imaging for Soft Tissue and Osseous Reconstruction in the Head and Neck. Clin. Plast. Surg. 12:279, 1985.

Taylor, R., Cutting, C., Kim, Y., Kalvin, A., Larose, D., Haddad, B., Khoramabadi, D., Noz, M., Olyha, R., Bruun, N., Grimm, D.: Model-Based Optimal Planning and Execution System with Active Sensing and Passive Manipulation for Augmentation of Human Precision in Computer-Integrated Surgery. Proc. 2nd Int. Symp.

Experimental Robotics, Toulouse, France, June 25-27, 1991a. Springer Verlag, In press.

Taylor, R., Paul, H., Cutting, C., Mittelsadt, B., Hanson, W., Kazanzides, P., Musits, B., Kim, Y., Kalvin, A., Haddad, B., Khoramabadi, D., LaRose, D.: Augmentation of human precision in computer-integrated surgery. Informatique at Technologie en Biologie at a Medicine, 1991b. In press.

Udupa, J. and Odhner, D.: Interactive surgical planning: High speed object rendition and manipulation without specialized hardware. Proc. First IEEE Conf. Visualiz. in Biomed. Comput. 1:330, 1990. 